\title{
Diagnóstico da cria e recria de bezerras em propriedades leiteiras no município de Corinto (MG)
}

\author{
[Survey of the pre-weaning and post-weaning of cow calves on dairy farms \\ in the municipality of Corinto $(M G)]$
}

\section{"Artigo Científico/Scientific Article"}

\author{
Tânia Guimarães Rabello Conceição ${ }^{1}$, Marcos Aurélio Lopes ${ }^{2 *}$, Milton Ghedini Cardoso ${ }^{2}$, Juliana \\ Aparecida Vieira ${ }^{3}$, Alessandro Botelho Pereira ${ }^{4}$
}

\author{
${ }^{1}$ EMATER-MG, Corinto-MG, Brasil. \\ ${ }^{2}$ Departamento de Medicina Veterinária, Universidade Federal de Lavras, Lavras-MG, Brasil. \\ ${ }^{3}$ Departamento de Zootecnia, Universidade Federal de Lavras, Lavras, MG, Brasil. \\ ${ }^{4}$ Empresa de Pesquisa Agropecuária de Minas Gerais, EPAMIG, Lavras, MG, Brasil. \\ *Autor para correspondência/Corresponding author: E-mail: malopes@ dmv.ufla.br
}

\begin{abstract}
Resumo
Objetivou-se realizar um diagnóstico de propriedades produtoras de leite, no que diz respeito a aspectos relacionados à cria e recria de fêmeas bovinas leiteiras, no município de Corinto, MG. Foram realizadas entrevistas com 30 produtores de leite de diferentes níveis tecnológicos e escalas de produção de leite, no período de abril a julho de 2016. Foi aplicado um formulário semi estruturado contendo 157 questões. Os dados foram cadastrados em planilhas do software Sphinx ${ }^{\circledR}$ e realizado o agrupamento das respostas por meio de sua categorização e frequência. Os resultados demonstraram pontos fortes, incluindo realização de cura de umbigo (93,3\%), localização dos bezerreiros acima (20\%), ao lado (46,7\%) ou longe do curral (10,0\%). Porém, diversas limitações no manejo da criação de bezerras e novilhas foram identificadas, como a inexistência de calendário sanitário $(83,3 \%)$, não realização de escrituração zootécnica $(60,7 \%)$, ordenha manual com bezerro ao pé $(63,3 \%)$, não execução da pesagem rotineira das bezerras $(96,7 \%)$, não fornecimento do leite de transição para as bezerras $(83,3 \%)$, além de possível falha no diagnóstico de doenças por parte dos produtores. Assim, é imperativo a disseminação de práticas de manejo adequadas pelos técnicos extensionistas e a formulação de políticas públicas que atendam as aspirações e promovam a sustentabilidade econômica e social dos agricultores familiares.
\end{abstract}

Palavras-chave: manejo; bezerra; novilha; agricultura familiar; pecuária de leite.

\begin{abstract}
The aim was to carry out a survey of dairy farms concerning aspects related to the pre-weaning and postweaning of dairy cows. Interviews were conducted with 30 dairy farmers of different technological levels and milk production scale in the period of April to July of 2016. A semi-structured form containing 157 questions was applied. The data were recorded in Sphinx ${ }^{\circledR}$ software spreadsheets and the grouping of the responses was conducted by means of their categorization and frequency. The results demonstrated strong points, including navel dipping (93.3\%), location of the calves above (20\%), close to $(46.7 \%)$ or far from the corral (10.0\%). However, several limitations in the management of the rearing of the heifers and calves were identified, such as the inexistence of a sanitary schedule (83.3\%), no zootechnical bookkeeping (60.7\%), hand milking with suckling calves $(63.3 \%)$, no routine calf weighing (96.7\%), no supply of transition milk to female calves (83.3\%), in addition to the possible failure of the farmers to diagnose diseases. Thus, the dissemination of adequate management practices by extension technicians and the formulation of public policies that meet the aspirations and promote the economic and social sustainability of family farmers are imperative.
\end{abstract}

Keywords: management; calf; heifer; family farming; dairy farming. 


\section{Introdução}

O estado de Minas Gerais é o maior produtor de leite do Brasil, com produção anual de 6,1 bilhões de litros, correspondendo a $64,4 \%$ de toda a produção da região sudeste e $26,3 \%$ do total da produção nacional (IBGE, 2016). O município de Corinto está localizado na região Central de Minas Gerais, com um rebanho bovino de 64.093 cabeças e produção anual de 55.661 mil litros de leite de vaca, sendo 30.923 vacas ordenhadas; portanto, produção média de 1.800 litros de leite/vaca/ano, enquanto a produtividade média nacional foi de 1.525 litros/vaca/ano (IBGE, 2014). No município a agricultura familiar desempenha um papel de grande importância social, detendo $70 \%$ dos estabelecimentos rurais, de acordo com o último senso agropecuário (IBGE, 2006).

$\mathrm{Na}$ bovinocultura leiteira muito se tem sofrido na reposição das matrizes, com a introdução de touros de corte no rebanho, objetivando bezerros (machos e fêmeas) para a produção de carne. Um dos principais fatores que desestimulam os produtores a produzir novilhas leiteiras para reposição é o elevado custo. Durante a fase de aleitamento ao primeiro parto (fases de cria e recria), os animais jovens não contribuem com a renda direta da atividade leiteira, sendo responsáveis por 15 a $20 \%$ do custo do litro de leite produzido (Mourits et al., 1997; Mourits et al., 1999). Dessa forma, é fundamental que o manejo da cria e recria seja adequado para que a mortalidade e as perdas econômicas sejam minimizadas e o crescimento otimizado, visando a antecipação da idade ao primeiro parto e, consequentemente, maior retorno econômico.

Estudos anteriores foram conduzidos para entender como os produtores criam os animais de reposição em algumas regiões do Brasil (Machado Neto et al., 2004; Hötzel et al., 2014; Santos e Bittar, 2015). No entanto, a despeito da expressiva participação da agricultura familiar na produção de leite do estado de Minas Gerais, as práticas adotadas por esses agricultores nas fases de cria e recria de fêmeas bovinas são pouco conhecidas. Diante disso, e considerando a importância do tema, objetivou-se, com este estudo, caracterizar os fatores associados à cria e recria de fêmeas bovinas de propriedades leiteiras em regime de economia familiar no município de Corinto, MG.

\section{Material e Métodos}

A abordagem da pesquisa é do tipo qualitativa (Bryman et al., 2008; Creswell, 2013) e o instrumento de coleta de dados consistiu na aplicação de um formulário semiestruturado, contendo 157 perguntas, adaptado de Lopes et al. (2016), feitas a 30 produtores do município de Corinto, região Central de MG, onde o clima é tropical de altitude, temperatura média anual de $22,4^{\circ} \mathrm{C}$, com pluviosidade média anual de $1.157 \mathrm{~mm}$. A pesquisa foi realizada entre os meses de abril e julho de 2016.

As questões foram divididas nos temas: cadastro do produtor e da propriedade, caracterização do rebanho, do sistema de produção de leite, manejo nutricional e infraestrutura para cria e recria, controle sanitário e qualidade do leite e manejo da ordenha. Foram utilizadas planilhas do software Sphinx ${ }^{\circledR}$ (Sphinx, 2011) para cadastrar as informações coletadas e realizado o agrupamento das respostas por meio de sua categorização e frequência, conforme Bardin (2003). Os dados categóricos foram compilados de acordo com sua categoria no formulário de diagnóstico obedecendo o tema a qual pertenciam.

Os resultados foram comparados por meio de análises descritivas, utilizando o aplicativo MS Excel $^{\circledR}$, e agrupados em tabelas, objetivando uma melhor apresentação, comparação e discussão (Lopes et al., 2004).

\section{Resultados e Discussão}

A maioria das fazendas $(96,7 \%)$ foi caracterizada por sistemas de criação em regime a pasto, enquanto que sistemas de criação semiconfinados representaram 3,3\% do total. No Brasil predominam sistemas de produção de leite a pasto e em muitas regiões a maior parte da produção concentra-se na época de chuvas (Silva et al., 2010). Os sistemas a pasto incluíram propriedades tradicionais compostas por animais mestiços, pastagens degradadas e suplementação restrita durante o ano inteiro. Por outro lado, sistemas semi-confinados se caracterizaram como sistemas melhorados, pastejo intensivo e suplementação concentrada durante o verão e suplementação volumosa e concentrada durante o inverno. A estratificação das fazendas pelo volume produzido de leite e área demonstrou predominância de pequena escala de produção, com 6,$7 ; 46,7 ; 33,3$ e $13,3 \%$ das propriedades produzindo menos de 100 ; 100 a 299; 300 a 499 e mais de 500 L/dia, respectivamente. $\mathrm{O}$ tipo de ordenha identificada foi majoritariamente manual e com bezerro ao pé, com percentuais de 53,3 e 63,3\%, respectivamente, 
evidenciando a baixa tecnificação dos sistemas de produção familiares.

$\mathrm{Na}$ criação de bezerros os sistemas observados foram a baia coletiva $(13,3 \%)$ e o piquete $(86,7 \%)$. Neste caso, é fundamental distribuir os bezerros de acordo com a faixa etária em lotes pequenos para garantir boa observação e minimizar a promiscuidade entre os animais (Coelho e Franzoni, 2012). Na fase de aleitamento, bezerreiros em que os animais ficam individualizados são os mais indicados por apresentarem menor taxa de mortalidade (Machado Neto et al., 2004). Os bezerreiros se localizaram predominantemente acima $(20 \%)$, ao lado $(46,7 \%)$ ou longe do curral $(10,0 \%)$, o que consiste em prática importante para diminuir o carreamento de bactérias para dentro do bezerreiro, aumentando o risco de contaminação e o desafio a afecções.

A escrituração zootécnica, que representa um fator limitante para a gestão dos sistemas de produção de leite, não é realizada em $66,7 \%$ das propriedades familiares (Tabela 1). A identificação segura dos animais é a base para todas as funções

Entre as práticas de manejo adotadas na criação de bezerras estão a cura de umbigo, a descorna e a remoção de tetas extranumerárias. Apesar da maioria dos produtores entrevistados realizarem a cura de umbigo (Tabela 2), 57,15\% deles executaram a antissepsia umbilical por 1 a 2 dias, prática que poderia ser estendida por pelo menos 3 dias consecutivos (Coelho e Franzoni, 2012). Em estudo anterior foi observada redução significativa do risco de onfalopatias quando os cuidados com cordão umbilical foram executados (Grover e Godden, 2011), no presente estudo, parte dos produtores que realizam a cura do umbigo $(28,6 \%)$ utilizaram produto inadequado (por exemplo, spray larvicida-repelente), o que pode levar a falhas no tratamento e, consequentemente, ocasionar onfalites, onfaloflebites, hérnias e miíases umbilicais, além de permitir a entrada de patógenos via ascendente.

A descorna de bezerros, considerada essencial para facilitar o manejo do rebanho e prevenir injúrias a outros animais e aos tratadores, tem sido largamente empregada nas criações de bovinos leiteiros (Phillips, 2002; Rushen et al., 2008). A descorna foi feita pela maioria dos os produtores $(93,3 \%)$, sendo em sua maioria nos animais com mais de 30 dias de vida (Tabela 2). Os entrevistados utilizaram exclusivamente os métodos de ferro quente $(28,6 \%)$ e ferro quente elétrico $(71,4 \%)$. Por outro lado, o procedimento de do sistema de manejo, que resultam em progressos zootécnicos, controle e economia da produção (Lopes, 1997). O uso do nome foi o principal método de identificação das bezerras nos rebanhos estudados (Tabela 1), o que pode estar atrelado ao pequeno tamanho dos rebanhos, uma vez que o brinco auricular convencional é o principal método de identificação em rebanhos maiores (Lopes et al., 2013). Outro fator limitante para o controle zootécnico consiste na ausência da pesagem rotineira das bezerras, identificada em $96,7 \%$ das propriedades deste estudo (Tabela 1). Consequentemente, não só o monitoramento do crescimento da cria e recria é prejudicado, mas também o agrupamento adequado dos lotes e estabelecimento do momento ideal para o primeiro serviço das novilhas. Adicionalmente, embora a idade tenha sido utilizada como o principal critério para o desaleitamento das bezerras neste estudo $(70,8 \%)$, o consumo diário de concentrado e o peso da bezerra têm sido recomendados (Greenwood et al., 1997; Jasper e Weary, 2002), visando melhor uniformização do lote de bezerras desaleitadas. remoção de tetas extranumerárias, cujo objetivo é reduzir a incidência de mastite na fase adulta devido representarem via adicional de contaminação de micro-organismos, além de dificultarem a ordenha e prejudicarem a estética (Auad et al., 2010), foi realizado em apenas uma propriedade $(3,3 \%)$.

A colostragem exerce papel fundamental para garantir a transferência passiva de imunoglobulinas às bezerras recém-nascidas, além de ser fonte de nutrientes e energia (Godden, 2008). Nos sistemas de produção de leite familiares deste estudo, foi observado que a colostragem não foi realizada corretamente. Na maior parte $(93,3 \%)$ das propriedades não houve monitoramento da quantidade ingerida de colostro após o nascimento (Tabela 2). Também foi observado que apenas $16,7 \%$ dos produtores forneceram leite de transição para as bezerras. Embora estudos demonstrem que a absorção de imunoglobulinas presentes no colostro através do epitélio intestinal para a circulação do neonato ocorra nas primeiras 24 horas após o nascimento (Weaver et al., 2000), o fornecimento do leite de transição pode dificultar a adesão de bactérias à parede intestinal, reduzindo a incidência de diarreias durante as primeiras semanas de vida (Azevedo et al., 2013), além de ser economicamente vantajoso (Lopes e Vieira, 1998). Ademais, nenhuma propriedade realizou a avaliação da qualidade do colostro e apenas uma 
congelou o colostro para situações de emergência, práticas largamente recomendadas para que a criação de animais jovens seja bem-sucedida (Pritchett et al., 1994; McGuirk e Collins, 2004), especialmente em pequenas propriedades leiteiras ( $<200$ L/dia), cuja susceptibilidade das bezerras às doenças é maior devido ao uso de colostro com baixa qualidade nutricional e microbiológica (Santos et al., 2017).

Os resultados mostraram, ainda, que a maioria $(93,3 \%)$ das propriedades utilizaram aleitamento natural. Os utensílios utilizados na alimentação das bezerras foram lavados com água e sabão em 93,3\% das propriedades e com água e cloro em $7,7 \%$. O fornecimento de leite de vacas em tratamento com antibióticos ocorreu em 83,3\% das propriedades (Tabela 3 ), fator que pode causar resistência de bactérias devido ao fornecimento de sub-doses de antibiótico a partir do leite de vacas tratadas, além da alta carga

No presente estudo, a prática do fornecimento de água a partir do primeiro dia de vida da bezerra ocorreu em $96,7 \%$ das propriedades. Já o concentrado foi oferecido a partir do primeiro dia em $37,9 \%$ das propriedades e entre o segundo ao décimo quinto dia nas demais (Tabela 4). O fornecimento precoce de água é importante pois afeta o consumo inicial de concentrado (Kertz et al., 1984). Por outro lado, o consumo de concentrado o mais cedo possível estimula o desenvolvimento do rúmen e viabiliza a desmama precoce das bezerras (Quigley et al., 1991).

A forragem foi disponibilizada para as bezerras a partir do primeiro dia de vida na maioria das propriedades (Tabela 4). O tipo de forragem oferecida às bezerras consistiu predominantemente em pasto na época das águas e cana de açúcar (Saccharum officinarum) na seca (Tabela 4). Apesar da ingestão de forragem durante a fase de aleitamento favorecer o desenvolvimento físico do rúmen (Hill et al., 2008), o perfil de fermentação alterado retarda o crescimento das papilas ruminais (Khan et al., 2011). Em contrapartida, estudos mais recentes têm apoiado o uso de forragem para bezerros durante a fase de aleitamento, como estratégia para aumento do consumo de concentrado, maior ganho de peso e redução de comportamentos anormais das bezerras (Castells et al., 2012; Terré et al., 2013; Imani et al., 2017), especialmente em sistemas de criação intensivos. Além disso, uma vez que a forma física da forragem pode influenciar o desenvolvimento do rúmen, a disponibilidade, a digestibilidade, bem como a percentagem de folhas são fatores importantes a serem observados para otimizar o desenvolvimento das bezerras (Castells et al., 2012; Montoro et al., 2013).

Quanto ao manejo sanitário das bezerras e novilhas, a maioria $(83,3 \%)$ dos entrevistados afirmou existir um calendário sanitário na propriedade. As vacinas utilizadas se restringiram a brucelose, raiva, febre aftosa, paratifo, clostridioses e botulismo (Tabela 5). Apesar de ser obrigatório a vacinação contra febre aftosa, 10\% das propriedades não a realizam. $\mathrm{O}$ critério para o controle de carrapatos se baseou, principalmente, no grau de infestação $(63,3 \%)$, sendo que duas propriedades realizam o teste de biocarrapaticida, para determinação do produto para controle dos carrapatos (Tabela 5), o que pode estar relacionado ao desconhecimento e/ou ausência de assistência técnica por parte dos produtores. A desvermifugação foi realizada nas fases de aleitamento (86\%), após o desaleitamento $(76,7 \%)$ e na recria $(73,3 \%)$, sendo adotado, como critérios, intervalos fixos $(53,3 \%)$ ou visual $(46,7 \%)$. As doenças relatadas como mais prevalentes foram, em ordem decrescente, diarreia, tristeza parasitária, pneumonia e verminoses (Tabela 5). Um levantamento conduzido por Santos e Bittar (2015), em rebanhos comerciais dos estados de MG, PR e SP, mostrou que as principais doenças encontradas foram: diarreia (48\%), pneumonia $(22 \%)$, tristeza parasitária $(21 \%)$ e verminoses (5\%), dados semelhantes ao encontrado nesse levantamento. A diarreia tem sido apontada como a mais importante enfermidade de bezerras, sendo responsável pelo baixo desempenho e pelas altas taxas de mortalidade (Cho e Yoon, 2014). Em geral, acomete os animais nas três primeiras semanas de vida (Bendali et al., 1999; Svensson e Liberg, 2006) e entre diversos fatores de risco relacionados estão aleitamento natural, práticas de higiene precárias, presença de outras espécies animais na fazenda, elevada densidade de bezerros e ocorrência de doenças respiratórias (Frank e Kaneene, 1993; Bendali et al., 1999; Svensson et al., 2003; Klein-Jöbstl et al., 2014). Além disso, foi detectado que apenas $53,3 \%$ das propriedades isolam rotineiramente as bezerras doentes (Tabela 5). De fato, existe estreita relação entre o ambiente onde os animais se encontram e a ocorrência de doenças (Drackley, 2008), de modo que os bezerros doentes são fonte de risco potencial para a 
saúde dos demais animais que compartilham a instalação.

A atividade leiteira é uma forma de exploração pecuária complexa. Por isso, deve ser administrada com profissionalismo e, entre tantas variáveis, a criação de bezerras e novilhas é fator essencial para o equilíbrio da atividade. Este estudo destaca a escassez de conhecimentos técnicos referentes às práticas de criação de animais jovens pelos agricultores familiares. Assim, a compreensão dos desafios na criação de bezerras e novilhas leiteiras fornece oportunidades de treinamento e capacitação, os quais contribuirão para disseminação de práticas de manejo adequadas e redução das taxas de mortalidade e morbidade animal. Por fim, poderá ajudar ainda na equidade social e garantia da sustentabilidade, ao incorporar os pequenos produtores de leite ao mercado, com exploração agropecuária de alta eficiência técnica e econômica.

Tabela 1. Caracterização do controle zootécnico utilizado para criação de bezerras das 30 propriedades estudadas no município de Corinto-MG (período de abril a julho de 2016).

\begin{tabular}{|c|c|c|c|}
\hline Questão & Averiguação & $\mathbf{n}$ & $\%$ \\
\hline \multirow[t]{2}{*}{ Realiza escrituração zootécnica? } & Sim & 10 & 33,30 \\
\hline & Não & 20 & 66,70 \\
\hline \multirow[t]{2}{*}{ Método de identificação das bezerras } & Brinco & 7 & 23,30 \\
\hline & Nome & 23 & 76,7 \\
\hline \multirow[t]{2}{*}{ Realiza pesagem das bezerras } & $\operatorname{Sim}$ & 1 & 3,30 \\
\hline & Não & 29 & 96,70 \\
\hline \multirow[t]{6}{*}{ Critério de desaleitamento* } & Peso & 2 & 8,30 \\
\hline & Idade & 17 & 70,80 \\
\hline & Na secagem da vaca & 1 & 4,20 \\
\hline & A vaca rejeita o bezerro & 2 & 8,30 \\
\hline & Diminui a produção & 1 & 4,20 \\
\hline & Pelo cruzamento da mãe & 1 & 4,20 \\
\hline \multirow[t]{5}{*}{ Com qual idade a bezerra é desaleitada? } & 4 a 6 meses & 3 & 10,00 \\
\hline & 7 a 9 meses & 15 & 50,00 \\
\hline & Acima de 9 meses & 8 & 26,60 \\
\hline & 60 dias antes da mãe parir & 2 & 6,70 \\
\hline & Até a mãe rejeitar & 2 & 6,70 \\
\hline \multirow[t]{4}{*}{ Critério para agrupamento da recria } & Sexo & 14 & 46,70 \\
\hline & Idade & 9 & 30,00 \\
\hline & Não separa & 6 & 20,00 \\
\hline & Outro & 1 & 3,30 \\
\hline \multirow[t]{4}{*}{ Idade a primeira cobertura } & Até 18 meses & 1 & 3,30 \\
\hline & 18 a 24 meses & 8 & 26,70 \\
\hline & 25 a 30 meses & 15 & 50,00 \\
\hline & Acima de 30 meses & 6 & 20,00 \\
\hline \multirow[t]{3}{*}{ Peso a primeira cobertura } & Até $299 \mathrm{~kg}$ & 6 & 20,00 \\
\hline & Acima de $300 \mathrm{~kg}$ & 23 & 76,70 \\
\hline & Não tem ideia & 1 & 3,30 \\
\hline
\end{tabular}

Em casos em que as frequências diferem do total dos casos: *Um produtor não respondeu à questão. 
Tabela 2. Caracterização da criação de bezerras das 30 propriedades estudadas da região de Corinto-MG (período de abril a julho de 2016).

\begin{tabular}{lccc}
\hline \multicolumn{1}{c}{ Questão } & Averiguação & n & \% \\
\hline Realiza curativo do umbigo? & Sim & 28 & 93,30 \\
Quantas vezes por dia é feito o curativo? & Não & 2 & 6,70 \\
& 1 vez & 25 & 89,30 \\
Quantos dias o umbigo é curado? & 2 vezes & 3 & 10,70 \\
& 1 dia & 12 & 42,85 \\
& 2 dias & 4 & 14,30 \\
O que usa para curar o umbigo? & 3 dias & 7 & 25,00 \\
& Até cair & 5 & 17,85 \\
Quanto tempo após o nascimento o & Spray & 8 & 28,60 \\
bezerro recebe colostro? & Iodo 10\% & 20 & 71,40 \\
Existe banco de colostro na propriedade? & 2 horas & 2 & 6,70 \\
& Mama sozinho & 28 & 93,30 \\
O que é feito com o leite de transição? & Sim & 1 & 3,33 \\
& Não & 29 & 96,66 \\
Quando é feita a descorna? & Outros bezerros & 5 & 16,70 \\
& Joga fora & 1 & 3,30 \\
& Usado para outros animais da fazenda & 24 & 80,00 \\
& Até 20 dias & 1 & 3,30 \\
& 30 dias ou mais & 16 & 53,30 \\
& Varia & 11 & 36,70 \\
\hline
\end{tabular}

Tabela 3. Caracterização das práticas de aleitamento de bezerras das 30 propriedades estudadas da região de Corinto-MG (período de abril a julho de 2016).

\begin{tabular}{|c|c|c|c|}
\hline Questão & Averiguação & n & $\%$ \\
\hline \multirow{2}{*}{ Como é oferecido o leite para o bezerro? } & Natural & 28 & 93,30 \\
\hline & Artificial com mamadeira & 2 & 6,70 \\
\hline \multirow{2}{*}{ Usa sucedâneo? } & Sim & 2 & 6,70 \\
\hline & Não & 28 & 93,30 \\
\hline \multirow{7}{*}{$\begin{array}{l}\text { Quantos litros de leite são fornecidos até } 30 \\
\text { dias? }\end{array}$} & 1 teta & 21 & 70,00 \\
\hline & Residual & 4 & 13,50 \\
\hline & 4 litros & 1 & 3,30 \\
\hline & 5 litros & 1 & 3,30 \\
\hline & $\begin{array}{l}\text { Aparta cedo e coloca para mamar as } \\
\qquad 12 \text { horas }\end{array}$ & 1 & 3,30 \\
\hline & Fica com a mãe até o meio dia & 1 & 3,30 \\
\hline & Mama antes da ordenha & 1 & 3,30 \\
\hline \multirow{7}{*}{ Entre 30 e 60 dias (litros) } & 1 teta & 15 & 51,75 \\
\hline & Residual & 9 & 31,00 \\
\hline & 4 litros & 1 & 3,45 \\
\hline & 5 litros & 1 & 3,45 \\
\hline & $\begin{array}{l}\text { Aparta cedo e coloca para mamar as } \\
\qquad 12 \text { horas }\end{array}$ & 1 & 3,45 \\
\hline & Fica com a mãe até o meio dia & 1 & 3,45 \\
\hline & Mama antes da ordenha & 1 & 3,45 \\
\hline \multirow{6}{*}{ Entre 60 e 90 dias (litros) } & Residual & 15 & 75,00 \\
\hline & 1 teta & 1 & 5,00 \\
\hline & 4 litros & 1 & 5,00 \\
\hline & 5 litros & 1 & 5,00 \\
\hline & $\begin{array}{l}\text { Aparta cedo e coloca para mamar as } \\
\qquad 12 \text { horas }\end{array}$ & 1 & 5,00 \\
\hline & Fica com a mãe até o meio dia & 1 & 5,00 \\
\hline \multirow{2}{*}{ Qual a temperatura do leite fornecido? } & Ambiente & 4 & 36,40 \\
\hline & Morno & 7 & 63,60 \\
\hline \multirow{2}{*}{ O aleitamento é feito logo após a ordenha? } & Sim & 7 & 87,50 \\
\hline & Não & 1 & 12,50 \\
\hline \multirow{2}{*}{$\begin{array}{l}\text { Recebe leite de vacas em tratamento com } \\
\text { antibióticos? }\end{array}$} & Sim & 25 & 83,30 \\
\hline & Não & 5 & 16,70 \\
\hline
\end{tabular}


Tabela 4. Caracterização das práticas de alimentação de bezerras das 30 propriedades estudadas da região de CorintoMG (período de abril a julho de 2016).

\begin{tabular}{l} 
Questão \\
\hline Com quantos dias recebe água pela primeira \\
vez? \\
Com quantos dias o bezerro recebe concentrado \\
pela primeira vez?
\end{tabular}

Fornece forragem para os bezerros?

Com quantos dias a bezerra recebe forragem pela primeira vez?

Se "outro", defina

Qual forragem é oferecida na época das águas?

Qual forragem é oferecida na época da seca?

\begin{tabular}{ccc} 
Averiguação & $\mathbf{n}$ & $\mathbf{\%}$ \\
\hline A partir do primeiro dia & 29 & 96,70 \\
Outro & 1 & 3,30 \\
A partir do primeiro dia & 11 & 36,67 \\
Após o quinto dia & 1 & 3,33 \\
8 dias & 1 & 3,33 \\
10 dias & 1 & 3,33 \\
A partir dos 10 dias & 1 & 3,33 \\
15 dias & 1 & 3,33 \\
30 dias & 5 & 16,70 \\
30 a 45 dias & 1 & 3,33 \\
40 dias & 1 & 3,33 \\
60 dias & 1 & 3,33 \\
3 meses & 1 & 3,33 \\
Na seca, desde o primeiro dia & 1 & 3,33 \\
Não recebe & 3 & 10,00 \\
Só animais mais sentidos & 1 & 3,33 \\
Sim & 30 & 100,00 \\
A partir do primeiro dia & 25 & 83,30 \\
Outro & 5 & 16,70 \\
15 dias & 1 & 20,00 \\
60 dias & 2 & 40,00 \\
Na seca & 1 & 20,00 \\
Quinto dia & 1 & 20,00 \\
Cana-de-açúcar & 1 & 3,30 \\
Pastagem & 29 & 96,70 \\
Feno & 1 & 3,30 \\
Capim picado & 5 & 16,70 \\
Silagem de milho & 1 & 3,30 \\
Pastagem & 5 & 16,70 \\
Cana-de-açúcar & 18 & 60,00 \\
\hline & & \\
& 1 & 1
\end{tabular}

Tabela 5. Caracterização do controle sanitário da criação de bezerras e novilhas das 30 propriedades estudadas no município de Corinto, MG (período de abril a julho de 2016).

Quais vacinas são aplicadas regularmente?

Critério para controle de carrapato

Realiza testes biocarrapaticida?

Frequência de realização do teste

Realização de vermifugação*

Critério adotado para tratamento

Quais doenças mais ocorrem nas bezerras?

Bezerras doentes são separadas das sadias?
Questão

\begin{tabular}{|c|c|c|}
\hline Averiguação & $\mathbf{n}$ & $\%$ \\
\hline Sim & 5 & 16,70 \\
\hline Não & 25 & 83,30 \\
\hline Brucelose & 30 & 100,00 \\
\hline Raiva & 20 & 66,70 \\
\hline Febre aftosa & 27 & 90,00 \\
\hline Paratifo & 1 & 3,30 \\
\hline Clostridioses & 29 & 96,70 \\
\hline Botulismo & 6 & 20,00 \\
\hline au de infestação & 19 & 63,30 \\
\hline Intervalo fixo & 3 & 10,00 \\
\hline dicação de bula & 8 & 26,70 \\
\hline Sim & 2 & 6,70 \\
\hline Não & 28 & 93,30 \\
\hline Anual & 1 & 50,00 \\
\hline carrapato não quer cair & 1 & 50,00 \\
\hline zerras mamando & 26 & 86,70 \\
\hline erras desmamadas & 23 & 76,70 \\
\hline Novilhas & 22 & 73,30 \\
\hline Não utiliza & 1 & 3,30 \\
\hline Observação & 14 & 46,70 \\
\hline Período fixo & 16 & 53,30 \\
\hline Diarreia & 14 & 46,70 \\
\hline Pneumonia & 3 & 10,00 \\
\hline isteza parasitária & 10 & 33,30 \\
\hline Verminoses & 1 & 3,30 \\
\hline Sim & 16 & 53,30 \\
\hline Não & 14 & 46,70 \\
\hline
\end{tabular}

Em casos em que as frequências diferem do total dos casos: *Um produtor não respondeu à questão. 


\section{Conclusão}

Os resultados desta pesquisa representam a realidade local das fazendas tradicionais da região e demonstram várias limitações que necessitam ser superadas ou minimizadas. Os seguintes pontos fortes podem ser destacados: realização de cura de umbigo, localização dos bezerreiros predominantemente acima, ao lado ou longe do curral. Por outro lado, deficiências no manejo da criação de bezerras e novilhas foram identificadas, incluindo a inexistência de calendário sanitário na propriedade, não realização de escrituração zootécnica, ordenha manual com bezerro ao pé, ausência de dimensionamento do rebanho, não execução da pesagem rotineira das bezerras e não fornecimento do leite de transição para as bezerras por parte dos produtores. Portanto, é imperativo a disseminação de práticas de manejo adequadas pelos técnicos extensionistas e a formulação de políticas públicas que atendam as aspirações e promovam a sustentabilidade econômica e social dos agricultores familiares.

\section{Conflito de Interesse}

Os autores declaram não existir conflito de interesse.

\section{Agradecimentos}

Os autores agradecem à EMATER - MG, por ter possibilitado a realização desta pesquisa, e ao CNPq pela concessão de bolsa de produtividade ao segundo autor.

\section{Referências}

Azevedo, R.A.; Araújo, L.; Coelho, S.G.; Faria Filho, D.E.; Duarte, E.R.; Geraseev, L.C. Desempenho de bezerros alimentados com silagem de leite de transição. Pesquisa Agropecuária Brasileira, 48: 545-552, 2013.

Bardin, L. Análise de conteúdos. 1 ed. Lisboa: Edições 70, 2003. 225p.

Bendali, F.; Sanaa, M.; Bichet, H.; Schelcher, F. Risk factors associated with diarrhoea in newborn calves. Veterinary Research, 30: 509-522, 1999.

Bryman, A.; Becker, S.; Sempik, J. Quality criteria for quantitative, qualitative and mixed methods research: a view from social policy. International Journal of Social Research Methodology, 11(4): 261-276, 2008.

Castells, L.; Bach, A.; Araujo, G.; Montoro, C.; Terre, M. Effect of different forage sources on performance and feeding behavior of Holstein calves. Journal of Dairy Science, 95: 286-293, 2012.

Cho, Y.; Yoon, K.J. An overview of calf diarrheainfectious etiology, diagnosis, and intervention. Journal of Veterinary Science, 15: 1-17, 2014.

Coelho, S.G.; Franzoni, A.P.S. Comportamento e bem-estar de bezerros na bovinocultura leiteira. Cadernos Técnicos de Veterinária e Zootecnia (UFMG), 67: 135-151, 2012.

Creswell, J.W. Designing a qualitative study. In: Qualitative inquiry and research design. London: Sage, 2013. p.42-68.

Drackley, J.K. Calf nutrition from birth to breeding. Veterinary Clinics Food Animal, 24: 55-86, 2008.

Frank, N.A.; Kaneene, J.N. Management risk factors associated with calf diarrhea in Michigan dairy herds. Journal of Dairy Science, 76: 1313-1323, 1993.

Godden, S. Colostrum management for dairy calves. Veterinary Clinics of North America: Food Animal Practice, 24: 19-39, 2008.

Greenwood, R.H.; Morrill, J.L.; Titgemeyer, E.C. Using dry feed intake as a Percentage of initial body weight as a weaning criterion. Journal of Dairy Science, 80: 2542-2546, 1997.

Grover, W.M.; Godden, S. Efficacy of a new navel dip to prevent umbilical infection in dairy calves. Bovine Practitioner, 45: 70-77, 2011.

Hill, T.M.; Bateman, H.G.; Aldrich, J.M.; Schlotterbeck, R.L. Effects of the amount of chopped hay or cottonseed hulls in a textured calf starter on young calf performance. Journal of Dairy Science, 91: 2684-2693, 2008.

Hötzel, M.J.; Longo, C.; Balcão, L.F.; Cardoso, C.S.; Costa, J.H.C. A Survey of management practices that influence performance and welfare of dairy calves reared in Southern Brazil. PLoS ONE, 9: 1-17, 2014.

Imani, M.; Mirzaei, M.; Baghbanzadeh-Nobari, B.; Ghaffari, M.H. Effects of forage provision to dairy calves on growth performance and rumen fermentation: A meta-analysis and metaregression. Journal of Dairy Science, 100: 1136-1150, 2017.

IBGE - Instituto Brasileiro de Geografia e Estatística. Censo agropecuário. 2006. Rio de Janeiro. Disponível em: <http://www.ibge.gov.br/home/estatistica/econ omia/agropecuaria/censoagro/>. Acesso em: 23 mar. 2017.

IBGE - Instituto Brasileiro de Geografia e Estatística. Pesquisa da Pecuária Municipal. 
2006. Brasília, DF. Disponível em: <http://www.sidra.ibge.gov.br>. Acesso em: 23 mar. 2017.

IBGE - Instituto Brasileiro de Geografia e Estatística. Produção da pecuária municipal. 2014. Disponível em: <http://biblioteca.ibge.gov.br/ visualizacao/periodicos/84/ppm_2014_v42_br. pdf>. Acesso em: 04 de jan. 2016.

IBGE - Instituto Brasileiro de Geografia e Estatística. Pesquisa trimestral do leite. 2016. Disponível em: < https://www.ibge.gov.br/estatisticasnovoportal/economicas/agricultura-epecuaria/9209-pesquisa-trimestral-doleite.html?\&t=series-historicas>. Acesso em: 08 fev. 2018.

Jasper, J.; Weary, D.M. Effects of ad libitum milk intake on dairy calves. Journal of Dairy Science, 85: 3054-3058, 2002.

Kertz, A.F.; Reutzel, L.F.; Mahoney, J.H. Ad libitum water intake by neonatal calves and its relationship to calf starter intake, weight gain, feces score, and season. Journal of Dairy Science, 67: 2964-2969, 1984.

Khan, M.A.; Weary, D.M.; Von Keyserlingk, M.A.G. Effects of milk ration on solid feed intake, weaning, and performance in dairy heifers. Journal of Dairy Science, 94: 10711081, 2011.

Klein-Jöbstl, D.; Iwersen, M.; Drillich, M. Farm characteristics and calf management practices on dairy farms with and without diarrhea: A case-control study to investigate risk factors for calf diarrhea. Journal of Dairy Science, 97: 5110-5119, 2014.

Lopes, M.A. Informática aplicada à bovinocultura. Jaboticabal: FUNEP, 1997. 82p.

Lopes, M.A.; Lima, A.L.R.; Carvalho, F.deM.; Reis, R.P.; Santos, I.C.; Saraiva, F.H. Controle gerencial e estudo da rentabilidade de sistemas de produção de leite na região de Lavras (MG). Ciência e Agrotecnologia, 28(4): 883-892, 2004.

Lopes, M.A.; Silva, M.D.; Demeu, A.A.; Gomide, D.R.; Bruhn, F.R.P. Custo da implantação e utilização de dois métodos de identificação de bovinos leiteiros. Revista Ceres, 60(6): 757764, 2013.

Lopes, M.A.; Reis, E.M.B.; Ferrazza, R.A. Formulário de diagnóstico da propriedade leiteira. Boletim Técnico. Lavras: UFLA, 2016. 19p.

Lopes, M.A.; Vieira, P.deF. Criação de bezerros leiteiros. FUNEP: Jaboticabal, 1998. 69p.

Auad, A.M.; Santos, A.M.B.dos; Carneiro, A.V.; Ribeiro, A.C.deC.L.; Carvalho, AdaC.; Freitas, A.F.de; et al. Manual de bovinocultura de leite. Brasília: LK Editora, 2010. 607p.

Machado Neto, R.; Faroni, C.E.; Pauletti, P.; Bessi, R. Levantamento do manejo de bovinos leiteiros recém-nascidos: desempenho e aquisição de proteção passiva. Revista Brasileira de Zootecnia, 33: 2323-2329, 2004.

Maynou, G.; Bach, A.; Terré, M. Feeding of waste milk to Holstein calves affects antimicrobial resistance of Escherichia coli and Pasteurella multocida isolated from fecal and nasal swabs. Journal of Dairy Science, 100: 2682-2694, 2017.

Mcguirk, S.M.; Collins. M. Managing the production, storage, and delivery of colostro. Veterinary Clinics North America, 20: 593603, 2004.

Montoro, C.; Miller-Cushon, E.K.; Devries, T.J.; Bach, A. Effect of physical form of forage on performance, feeding behavior, and digestibility of Holstein calves. Journal of Dairy Science, 96: 1117-1124, 2013.

Mourits, M.; Dijkhuizen, A.; Huirne, R.B.M.; Galligan, D.T. Technical and economic models to support heifer management decisions: Basic concepts. Journal of Dairy Science, 80: 14061415, 1997.

Mourits, M.; Huirne, C.; Dijkhuizen, A.A.; Kristensen, A.R.; Galligan, D.T. Economic optimization of dairy heifer management decisions. Agricultural Systems, 61: 17-31, 1999.

Phillips, C. Cattle behaviour and welfare. $2^{\text {nd }} \mathrm{ed}$. Wiley-Blackwell: Oxford, 2002. 274p.

Pritchett, L.C.; Gay, C.C.; Hancock, D.D.; Besser, T.E. Evaluation of the hydrometer for testing immunoglobulin G1 concentration in Holstein colostrums. Journal of Dairy Science, 77: 1761-1767, 1994.

Quigley, J.D.; Smith, P.; Heitmann, R.N. Changes in plasma volatile fatty acids in response to weaning and feed intake in young calves. Journal Dairy Science, 74: 258-263, 1991.

Rushen, J.; Passillé, A.M.; Von Keyserlingk, M.A.G.; Weary, D.M. The Welfare of Cattle. Springer: Netherlands, 2008. 310 p. 
Santos, G.; Bittar, C.M.M. A survey of dairy calf management practices in some producing regions in Brazil. Revista Brasileira de Zootecnia, 44(10): 361-370, 2015.

Santos, G.; Silva, J.T.; Santos, F.H.R.; Bittar, C.M.M. Nutritional and microbiological quality of bovine colostrum samples in Brazil. Revista Brasileira de Zootecnia, 46(1): 72-79, 2017.

Silva, J.J.; Carvalho, D.M.G.; Gomes, R.A.B.; Rodrigues, A.B.C. Produção de leite de animais criados em pastos no Brasil. Veterinária e Zootecnia, 17(1): 26-36, 2010.

Sphinx Brasil. Manual de uso do software: Sphinx rápido. $2^{\text {nd }}$ ed. Sphinx: Canoas, 2011. $47 \mathrm{p}$.

Svensson, C.; Liberg, P. The effect of group size on health and growth rate of Swedish dairy calves housed in pens with automatic milk- feeders. Preventive Veterinary Medicine, 73: 43-53, 2006.

Svensson, C.; Lundborg, K.; Emanuelson, U.; Olsson, S.O. Morbidity in Swedish dairy calves from birth to 90 days of age and individual calflevel risk factors for infectious diseases. Preventive Veterinary Medicine, 58: 179197, 2003.

Terré, M.; Pedrals, E.; Dalmau, A.; Bach, A. What do preweaned and weaned calves need in the diet: A high fiber contente or a forage source? Journal of Dairy Science, 96: 5217-5225, 2013.

Weaver, D.M.; Tyler, J.W.; Vanmetre, D.C.; Hostetler, D.E.; Barrington, G.M. Passive transfer of colostral immunoglobulins in calves. Journal of Veterinary Internal Medicine, 14: 569-577, 2000. 